\title{
Role of pre-ordered liquid in the selection mechanism of crystal polymorphs during nucleation
}

Cite as: J. Chem. Phys. 153, 104508 (2020); https://doi.org/10.1063/5.0017575

Submitted: 08 June 2020 . Accepted: 23 August 2020 . Published Online: 11 September 2020

(iD) Sarath Menon, (D) Grisell Díaz Leines, (iD) Ralf Drautz, and (i) Jutta Rogal
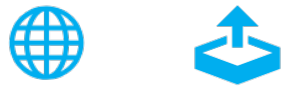

\section{ARTICLES YOU MAY BE INTERESTED IN}

Connection between liquid and non-crystalline solid phases in water

The Journal of Chemical Physics 153, 104503 (2020); https://doi.org/10.1063/5.0018923

Identification of a multi-dimensional reaction coordinate for crystal nucleation in $\mathrm{Ni} 3 \mathrm{Al}$ The Journal of Chemical Physics 152, 224504 (2020); https://doi.org/10.1063/5.0010074

Structure and dynamics of the Lennard-Jones fcc-solid focusing on melting precursors The Journal of Chemical Physics 153, 104506 (2020); https://doi.org/10.1063/5.0015371

\section{Challenge us.} What are your needs for periodic signal detection?

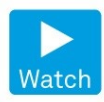

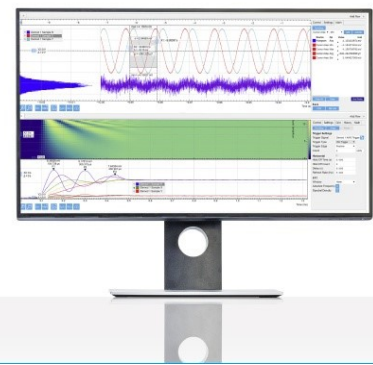

- Zurich Instruments 


\title{
Role of pre-ordered liquid in the selection mechanism of crystal polymorphs during nucleation
}

\author{
Cite as: J. Chem. Phys. 153, 104508 (2020); doi: 10.1063/5.0017575 \\ Submitted: 8 June 2020 - Accepted: 23 August 2020 • \\ Published Online: 11 September 2020
}

Sarath Menon, (D) Grisell Díaz Leines, (D) Ralf Drautz, (D) and Jutta Rogal ${ }^{\mathrm{a})}$ (D)

AFFILIATIONS

Interdisciplinary Centre for Advanced Materials Simulation, Ruhr-Universität Bochum, 44801 Bochum, Germany

\author{
a) Author to whom correspondence should be addressed: jutta.rogal@rub.de
}

\begin{abstract}
We investigate the atomistic mechanism of homogeneous nucleation during solidification in molybdenum employing transition path sampling. The mechanism is characterized by the formation of a pre-structured region of high bond-orientational order in the supercooled liquid followed by the emergence of the crystalline bulk phase within the center of the growing solid cluster. This precursor plays a crucial role in the process as it provides a diffusive interface between the liquid and crystalline core, which lowers the interfacial free energy and facilitates the formation of the bulk phase. Furthermore, the structural features of the pre-ordered regions are distinct from the liquid and solid phases and preselect the specific polymorph that nucleates. The similarity in the nucleation mechanism of Mo with that of metals that exhibit different crystalline bulk phases indicates that the formation of a precursor is a general feature observed in these materials. The strong influence of the structural characteristics of the precursors on the final crystalline bulk phase demonstrates that for the investigated system, polymorph selection takes place in the very early stages of nucleation.
\end{abstract}

Published under license by AIP Publishing. https://doi.org/10.1063/5.0017575

\section{INTRODUCTION}

Polymorph selection during crystallization is one of the most fundamental processes that play an important role in several applications, ranging from the development of pharmaceutical drugs to the design of novel metallurgical materials. The final bulk polymorph of a material may already be largely determined in the early stages of crystallization, and thus, fundamental knowledge of the nucleation process on the atomic scale is essential. Nevertheless, as many systems exhibit complex mechanisms of crystallization, characterized by multiple steps and the competition of various crystalline structures within small clusters, fundamental understanding of crystal nucleation processes and polymorph selection mechanisms remains elusive.

Classical nucleation theory $(\mathrm{CNT})^{2,3}$ provides a successful phenomenological description of homogeneous nucleation, but because of the simplifying approximations and its application on the mesoscale, CNT cannot capture quantitative details of nucleation mechanisms. A main assumption of CNT, the well-known capillarity approximation, is that small, spherical clusters are in the same thermodynamic phase as the bulk and have a sharp interface with the surrounding liquid. However, corrections to the interfacial free energy term that take into account the shape of the crystalline clusters ${ }^{4,5}$ and the finite size of a diffusive interface $^{6,7}$ are often needed to match experimental data for timescales and activation energies. Furthermore, in contrast to the assumptions within CNT, the formation of polymorphic clusters with intermediate phases that differ from the final bulk structure has been observed in a vast number of examples, ${ }^{1}$ even for simple model systems such as Lennard-Jones fluids ${ }^{8-10}$ and hard spheres.

Recently, several studies have reported non-classical nucleation mechanisms characterized by the initial formation of pre-ordered regions in the liquid that act as precursors of the crystallization and the selected polymorphic structures. ${ }^{12-19}$ The clusters of the prestructured liquid are regions of either increased bond-orientational $\operatorname{order}^{12,13,15,20}$ or density ${ }^{21-24}$ that promote the emergence of crystallites within the center of the clusters by reducing the interfacial free energy. ${ }^{13,25}$ The observation of pre-ordered regions in the melt has raised great interest in understanding the connection between 
structural and dynamical heterogeneity of the liquids and crystallization mechanisms. ${ }^{25-29}$ Russo et al. recently showed for model liquids that the structural differences between liquids and crystals indeed control their glass-forming or crystal-forming ability by suppressing or promoting the formation of crystalline precursors via a thermodynamic interface penalty. ${ }^{25}$ Furthermore, a structural analysis of pre-ordered liquid regions in hard spheres ${ }^{13}$ showed that the structural features of these regions resemble the coordination polyhedra of the crystalline structures formed in the growing clusters and, therefore, pre-determine the polymorphs selected during crystallization. Previously, we have shown that during solidification in the face-centered cubic ( $\mathrm{fcc}$ ) metal nickel, the pre-ordered liquid region plays an essential role in the structural description of the growing nucleus and its interfacial free energy and, thus, represents an order parameter that significantly enhances the reaction coordinate. ${ }^{19}$ Moreover, in agreement with previous findings for hard sphere models, these regions of higher bond-orientational order than the liquid predetermine the coordination of the fcc-hexagonal closed packed (hcp) polymorphs selected in $\mathrm{Ni}$, acting as precursors of the crystallization. However, questions remain whether this crystallization mechanism is generally observed in other metallic systems that exhibit different thermodynamically stable phases, and how the different structural natures of the pre-ordered liquid determines the polymorph selected in the growing solid nucleus.

In this work, we take a step further to address these questions and investigate the nucleation process during solidification in a body-centered cubic (bcc) metal, molybdenum, using transition path sampling (TPS) simulations. ${ }^{30,31}$ Molybdenum is widely used as a component in steel alloys as it improves corrosion resistance and weldability. ${ }^{32}$ The nucleation process in Mo is largely unknown, partially due to the high melting point (2896 K) that hampers experimental studies. In bcc metals, only a few theoretical studies exist, which focus on molecular dynamics (MD) simulations of rapid solidification during quenching in iron ${ }^{33-35}$ and zirconium, ${ }^{36}$ where it was observed that icosahedral short-range order in the liquid gradually transforms into bcc-like short-range order during nucleation without the formation of other competing phases. Likewise, Wang et al. ${ }^{34}$ showed that the regions of bcc-like short-range order yield the formation of bulk bcc during crystallization in Fe. However, due to the rare event nature of nucleation events, straightforward MD simulations are limited to extremely high cooling rates that can result in trajectories that strongly differ from the actual mechanism of crystallization at moderate undercoolings. Here, we use the statistical path ensembles obtained from TPS to analyze the nucleation mechanism and kinetics in Mo at moderate undercoolings. We show that, similar to our findings in $\mathrm{Ni}^{18,19}$ the initial formation of pre-ordered regions with increased bond-orientational order within the liquid promotes the emergence of the crystalline phase within the core of these clusters, acting as a precursor of crystallization. At different undercoolings, the structural characteristics of the pre-ordered regions appear similar, whereas the frequency of formation and extent of pre-ordered regions is more pronounced at larger undercoolings. Our results suggest that the structural heterogeneity of the undercooled liquid, characterized by differences between regions with low and high bond-orientational order, is directly linked to crystal nucleation in Mo. The strong spatial correlation between regions of high bond-orientational order in the liquid and critical fluctuations also implies that the structure of the undercooled liquid is, indeed, decisive for the nucleation mechanism. We use three different interatomic potentials for Mo and find that for two of the potentials the overall nucleation mechanism is essentially the same. The third potential yields a largely different structure of the undercooled liquid and does not crystallize. It appears to be important that the interatomic potentials correctly capture the structural features of both, the solid and the liquid phases, further corroborating the relation between the structural heterogeneity of the liquid and crystallization. With an extensive analysis of the Voronoi polyhedra (VP) found in the pre-structured liquid regions in Mo, we show that the structural features of these regions are inherently different from those found in the crystalline precursors of fcc metals. The correspondence between pre-structured regions within the liquid and locally favored bcc-like ordering strongly indicates that the precursors predetermine the final bulk phase. In these materials, the selection of the bulk polymorph, thus, takes place in the early stages of the crystallization process.

\section{COMPUTATIONAL APPROACH}

\section{A. Simulation setup}

To investigate the initial stages of nucleation and growth during solidification in molybdenum, we employ transition interface sampling (TIS). ${ }^{37}$ TIS is a variant of transition path sampling (TPS $)^{30}$ in which an ensemble of trajectories is created that connects two (meta-)stable states in phase space. In the current study, the two states of interest correspond to the liquid and solid phases in Mo. The ensemble of pathways is sampled with a Monte Carlo (MC) procedure in trajectory space, and the analysis of the path ensemble provides access to both kinetic and thermodynamic properties of the transition.

As an order parameter [or collective variable (CV)] to distinguish between the solid and liquid state, we use the size of the largest cluster consisting of solid particles, $n_{s}$, introduced in Sec. II B. The same order parameter is also used to define the positions of the interfaces in the TIS simulations. The TIS path ensembles are subsequently reweighted to obtain an estimate of the complete ensemble $e^{38,39}$ that contains each trajectory with its correct weight to represent unbiased simulations. It is then possible to project the reweighted path ensemble (RPE) into different low-dimensional CV spaces and compute quantities such as the free energy or averaged committor along arbitrary $C V s .{ }^{39}$ The free energy, $F$, in a $m$ dimensional CV space $\mathbf{q}=\left\{q_{1}(\mathbf{x}), \ldots, q_{m}(\mathbf{x})\right\}$, where $\mathbf{x}$ is a point in phase space, is, for example, given by

$$
F(\mathbf{q})=-k_{\mathrm{B}} T \ln \rho(\mathbf{q})+\text { const., }
$$

with the Boltzmann constant $k_{\mathrm{B}}$, the temperature $T$, and the probability density $\rho(\mathbf{q})$, which is directly obtained from the reweighted path ensemble (RPE),

$$
\rho(\mathbf{q})=C \int \mathscr{D} \mathbf{x}^{L} \mathscr{P}\left[\mathbf{x}^{L}\right] \sum_{k=0}^{L} \delta\left(\mathbf{q}\left(\mathbf{x}_{k}\right)-\mathbf{q}\right) .
$$

Here, $\mathscr{P}\left[\mathbf{x}^{L}\right]$ is the RPE, $\int \mathscr{D} \mathbf{x}^{L}$ denotes the integral over all paths of length $L, \mathbf{x}^{L}=\left\{\mathbf{x}_{0}, \ldots, \mathbf{x}_{L}\right\}$, where $\mathbf{x}_{i}$ are slices along the path, 
$\delta(\mathbf{z})=\prod_{i=1}^{m} \delta\left(z_{i}\right)$ is the Dirac delta function, and $C$ is a normalization constant.

All molecular dynamics (MD) trajectories sampled in the TIS simulations were generated using the Large-scale Atomic/Molecular Massively Parallel Simulator (LAMMPS) ${ }^{40}$ code. The simulation box contained $N=4394$ atoms, which were found to be sufficient to minimize the finite size effects. To describe the interactions between Mo atoms, an embedded atom method (EAM) potential ${ }^{41}$ was used. The melting temperature of this potential was determined with the $\mathrm{z}$ method $^{42}$ to be $T_{m}=3472 \mathrm{~K}$. All TIS simulations were performed in the isothermal-isobaric (NPT) ensemble using a Nosé-Hoover thermostat and barostat as implemented in LAMMPS with an integration time step of $\Delta t=2 \mathrm{fs}$. The temperature was set to $2592 \mathrm{~K}$, $2708 \mathrm{~K}$, and $2782 \mathrm{~K}$, corresponding to $25 \%, 23 \%$, and $20 \%$ undercooling, respectively, at a pressure of $P=0$. The TIS simulations were performed using a python wrapper ${ }^{43}$ with LAMMPS as the MD driver. The MC moves to create new trajectories during the simulations include shooting (65\%) as well as exchange between interface ensembles (35\%) to improve ergodicity of the sampling. ${ }^{44}$ Trajectories were recorded every five MC steps to ensure sufficient decorrelation. Along the $\mathrm{MD}$ trajectories, configurations were stored every $100 \mathrm{fs}$ at $25 \%$ undercooling and every $200 \mathrm{fs}$ at $23 \%$ and $20 \%$ undercooling. For each interface, a total number of 900 trajectories were included in the path ensemble.

\section{B. Structure analysis}

A key component in the analysis of the simulation results is the identification of the local structure around each atom. As a first step, we need to distinguish if an atom is in a liquid or solid environment. Here, we use a criterion that determines the structural correlation of each atom with its neighbors based on the Steinhardt bond order parameters. ${ }^{45,46}$ Two atoms are considered to be neighbors if they are within a specified cutoff distance from each other. The cutoff distance is set to $r_{\mathrm{c}}=3.6 \AA$ based on the first minima in the radial distribution function of all the solid phases and the liquid. A solid bond between the two atoms $i$ and $j$ exists if the correlation $s_{i j}=\sum_{m=-6}^{m=6} q_{6 m}(i) q_{6 m}^{*}(j)>0.5$, where $q_{l m}$ are the complex vectors of the spherical harmonics. As a second criterion, we determine the average correlation over the nearest neighbors $^{18,47}\left\langle s_{i}\right\rangle=1 / N_{\mathrm{nn}} \sum_{j=1}^{N_{\mathrm{nn}}} s_{i j}$, which refines the classification of solid atoms at the solid-liquid interface. An atom is considered as solid if it has more than seven solid bonds and if $\left\langle s_{i}\right\rangle>0.6$. Using a clustering algorithm, we can then identify clusters of solid atoms, where $n_{s}$ represents the number of atoms in the largest solid cluster.

Specific crystal structures are assigned to solid atoms using the averaged local bond order parameters, ${ }^{48} \bar{q}_{4}$ and $\bar{q}_{6}$. The corresponding reference map for Mo at $20 \%$ undercooling is shown in Fig. 1. To compute the reference maps, MD simulations were performed in the NPT ensemble for bcc, fcc, and hcp bulk structures as well as for the liquid phase for $3 \mathrm{~ns}$ at each undercooling. The simulation box contained 6912 atoms for fcc and hcp, and 3456 atoms for bcc and liquid. For each reference structure, 300 configurations were randomly chosen from the MD trajectories and the $\bar{q}_{4}$ and $\bar{q}_{6}$ values were calculated using the PYsCAL library. ${ }^{49}$ We calculate the overlap between bcc, hcp, and liquid distributions to be $\sim 10^{-5}$; hence, an atom is assigned to a particular reference

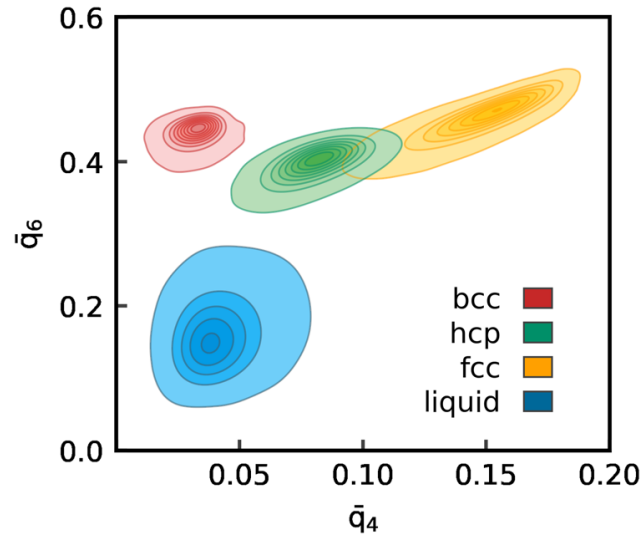

FIG. 1. Probability distribution of the averaged local bond order parameters $\bar{q}_{4}$ and $\bar{q}_{6}$ for bcc (red), hcp (green), fcc (yellow), and liquid (blue) in Mo at $20 \%$ undercooling. A darker shade of the colors indicates a higher probability density.

structure if the probability from the $\bar{q}_{4}-\bar{q}_{6}$ distribution is larger than this value. This selected cutoff probability allows for distinction between bcc, hcp, and liquid with a confidence of $98 \%$. A solid particle that does not fall into the region of any of the crystalline structures on the $\bar{q}_{4}-\bar{q}_{6}$ map is defined as pre-ordered. The preordered regions exhibit a local order greater than that of the liquid but no long range ordering, with $\bar{q}_{6}$ and $\bar{q}_{4}$ values that lie in between the liquid and the crystalline phases. The identification of the local order is challenging, ${ }^{50}$ especially at solid-liquid interfaces. Thus, we carefully verified that the identification of pre-ordered regions is not just an effect of the local order at the solid-liquid interface (see the supplementary material for details), but rather a region with distinct structural features, which are discussed in detail in Sec. III B.

\section{NUCLEATION AND GROWTH IN UNDERCOOLED MO}

\section{A. Mechanism of nucleation}

A basic step in investigating the nucleation mechanism is the analysis of the structural composition of the growing solid cluster, which can be directly extracted from the TIS path ensemble. The structural composition of the largest solid cluster, $n_{s}$, as an average over 600 trajectories connecting the liquid and solid state at $20 \%$ undercooling, is shown in Fig. 2(a). Similarly, the structural evolution was analyzed for $23 \%$ and $25 \%$ undercooling. For all undercoolings, the largest solid cluster is composed of more than $80 \%$ pre-structured particles up to approximately half of the critical cluster size. Subsequently, bcc emerges while the pre-ordered particles continue to constitute a significant fraction of the cluster. Both fcc and hcp do not play any noticeable role during the entire nucleation process in Mo. We further determine the spatial distribution of different crystal structures within the critical solid clusters, that is, at the transition state, for different undercoolings. The distribution at $20 \%$ undercooling $\left(n_{s}^{*}=172\right)$ averaged over 300 configurations is shown in Fig. 2(b). Here, $r_{\text {skin }}$ indicates the minimum distance to the surface of the solid cluster. The surface is defined by the atoms 
(a)

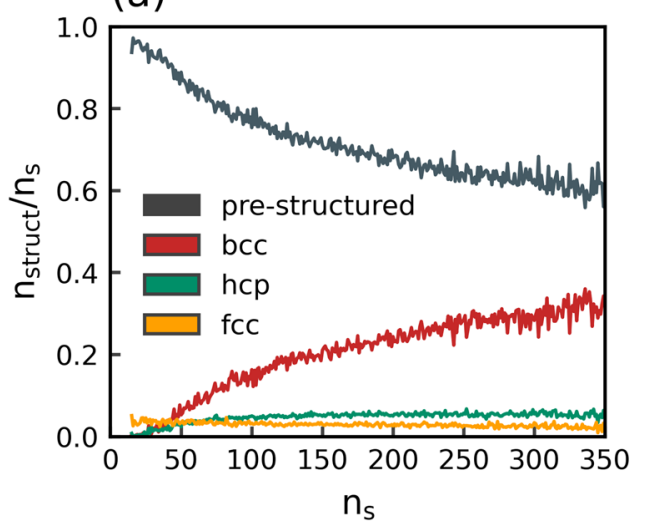

(b)

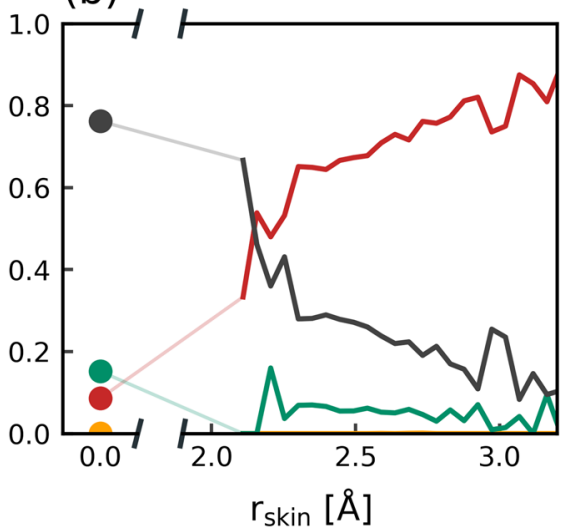

(c)

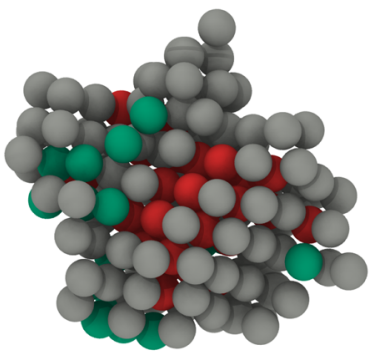

FIG. 2. (a) Average structural composition of the growing nucleus at $20 \%$ undercooling. (b) Fraction of crystalline structures and pre-ordered liquid as a function of the minimum distance to the surface of the solid cluster, $r_{\text {skin }}$, for critical nuclei at $20 \%$ undercooling. There are no data at distances up to $\approx 2 \AA$ from the surface atoms as this corresponds to the thickness of the surface layer. Transparent lines are a guide to the eye. (c) Snapshot of a typical configuration of the critical nuclei.

in the solid cluster that have at least one liquid neighbor. At the surface, where $r_{\text {skin }}=0 \AA$, the solid cluster is predominantly composed of pre-ordered liquid particles, whereas the core region consists of mainly bcc. A representative snapshot of a critical nucleus is shown in Fig. 2(c). The nucleation mechanism in Mo, thus, comprises the formation of pre-structured regions followed by the emergence of the thermodynamically stable, bcc bulk phase within the centers of these regions. For all three undercoolings studied in this work, the overall mechanism remains the same, except for small differences in the fraction of bcc that slightly decreases at higher undercoolings. This is predominantly due to kinetic effects, where shorter timescales at larger undercoolings hinder the rearrangement into the thermodynamically stable phase. Similar nucleation mechanisms have also been observed in the fcc metals $\mathrm{Al}^{17}$ and $\mathrm{Ni}^{19}$ exhibiting the formation of pre-structured liquid regions followed by the emergence of a crystalline core. The evolution of the structural composition in these fcc metals is, however, different, as they exhibit random hcp stacking together with fcc; whereas in bcc Mo, other crystalline structures do not play a role.

In addition to the analysis of the structural evolution during nucleation, we assess the free energy profile projected from the RPE according to Eq. (1). In Fig. 3(a), the blue line represents the free energy as a function of the largest solid cluster, $n_{s}$, at $20 \%$ undercooling. The nucleation barrier is extracted from the maximum along the free energy profile, yielding $4.4 \mathrm{eV}$ at $20 \%$ undercooling. The barrier decreases to $3.7 \mathrm{eV}$ and $2.1 \mathrm{eV}$ at $23 \%$ and $25 \%$ undercooling, respectively. Furthermore, the nucleation rates can directly be computed from the TIS ensemble. The associated timescales for nucleation vary from microseconds to nanoseconds with increasing undercooling (details concerning rates and barriers are given in the supplementary material). The decrease in free energy barriers and increase in nucleation rates at higher undercoolings are in qualitative agreement with CNT.

The initial formation of a pre-structured region in the liquid has a significant impact on the nucleation barrier. If we project the free energy along the number of bcc particles in the largest (a)

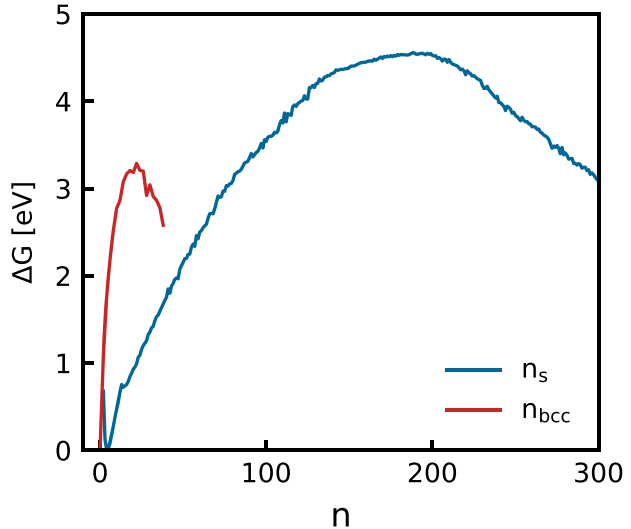

(b)

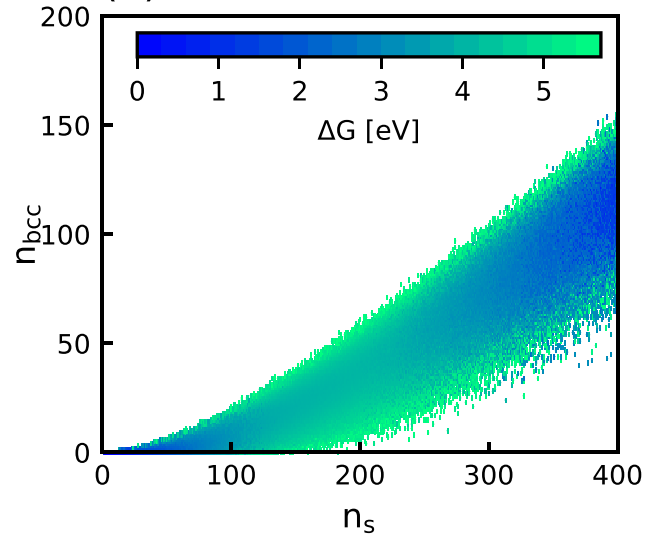

FIG. 3. (a) Free energy profiles projected from the reweighted path ensemble onto the size of the largest cluster, $n_{s}$ (blue), and on the number of bcc atoms in the largest cluster, $n_{\mathrm{bcc}}$ (red), for $20 \%$ undercooling. (b) Two-dimensional free energy projection onto $n_{s}$ and $n_{\mathrm{bcc}}$. 
cluster, $n_{\mathrm{bcc}}$ (which would be the parameter of choice within the capillarity assumption of $\mathrm{CNT}$ ), the barrier decreases by more than $1 \mathrm{eV}$; see the red line in Fig. 3(a). In addition, the critical nucleus size decreases dramatically. This is due to the fact that a projection onto $n_{\mathrm{bcc}}$ cannot capture the initial pre-ordering in the liquid, which subsequently serves as a precursor for the formation of bcc. The two-dimensional free energy projection onto $n_{s}$ and $n_{\text {bcc }}$ shown in Fig. 3(b) further supports our interpretation. Up to half of the critical cluster size, during the initial stage of the nucleation, $n_{\mathrm{bcc}}$ is close to zero indicating the absence of bcc particles in the cluster. Consequently, the initial step is dominated by the clustering of pre-structured particles, accompanied by an increase in the free energy of about $1 \mathrm{eV}$. When projecting only onto the number of bcc atoms, $n_{\mathrm{bcc}}$, this initial increase due to pre-ordering is not captured, leading to an overall smaller nucleation barrier. The same effect was observed for all undercoolings studied in this work.

The initial stage of pre-ordering has an important role in the nucleation mechanism as it acts as a diffusive interface between the liquid and crystal core, facilitating crystal nucleation, and, thus, acting as a precursor. This is similar to the findings for fcc $\mathrm{Ni}^{19}$ where including the pre-ordered region in the CV was necessary to accurately determine the free energy barrier. Comparing the nucleation mechanisms in bcc Mo and fcc Ni, we observe strong similarities: the initial step is characterized by the formation of a pre-ordered liquid state that acts as a precursor and is already associated with an increase in the free energy. A crystalline core emerges within that region surrounded by an interface layer of pre-structured liquid that persists throughout the nucleation process. However, the structure of the pre-ordered liquid is inherently different in the two systems, as discussed in Sec. III B.

\section{B. Structural features of the pre-ordered liquid}

The pre-ordered liquid state observed during the nucleation is not just a short-lived fluctuation in the liquid, but a mesocrystal phase with a structure that is different from both the liquid and the bulk phase. The structural characteristics of the pre-ordered liquid can already point toward the crystalline bulk phase that will emerge from it. Three aspects are of particular interest: the density, the crystallinity, and the local coordination polyhedra.

\section{Density}

The atomic density is obtained from the inverse of the Voronoi volume computed using Voro++. ${ }^{51}$ At all undercoolings, the difference in average density between the solid and the liquid is small (0.003 atom $/ \AA^{3}$ at $20 \%-25 \%$ undercoolings), as expected for a metallic bcc system. In Fig. 4, the average atomic density of the prestructured particles in the largest solid cluster is shown as a function of $n_{s}$ extracted from 600 liquid to solid trajectories at $20 \%$ undercooling. For each atomic configuration with a particular value of $n_{s}$, pre-structured atoms that belong to the solid cluster with at least two other pre-structured particles as neighbors are included in the calculation of the density. Results for other undercoolings are comparable. The density of the pre-ordered liquid particles is slightly larger than the one of the liquid indicating that the pre-ordered regions are more compact and distinct from the liquid phase. Since

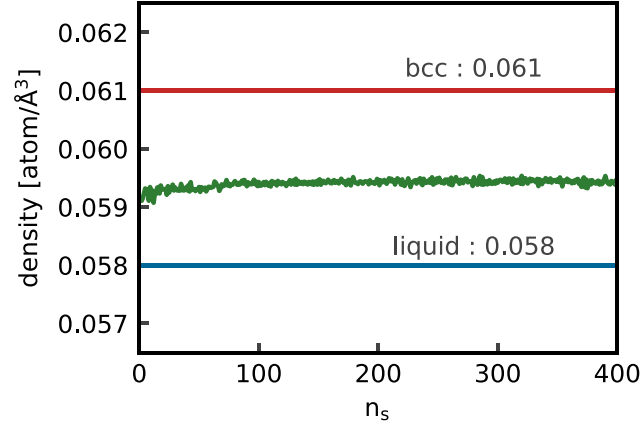

FIG. 4. Average atomic density of the pre-structured particles (green) as a function of $n_{s}$, together with the inverse volumes of bcc (red) and liquid (blue)

no change in the density of the pre-structured region is observed as the nucleation progress, the density itself does not appear to drive the transformation process. The formation of a precursor can be triggered by fluctuations either in the density ${ }^{21-24}$ or the bond orientational order. ${ }^{12,13,15,20}$ In the case of Mo, the small change in density can be considered rather as a result than a cause of the pre-ordering.

\section{Crystallinity}

The degree of orientational order in the pre-structured liquid is evaluated by computing the averaged bond order parameters $\bar{q}_{4}$ and $\bar{q}_{6}$. In Fig. 5, the distribution of pre-ordered particles on the $\bar{q}_{4}-\bar{q}_{6}$-map is presented averaged over 300 configurations each with pre-critical, critical, and post-critical cluster sizes at $20 \%$ undercooling. The analysis at $23 \%$ and $25 \%$ undercooling yields similar results. The distributions occupy the region between the liquid and the crystalline (bcc, hcp, and fcc) phases, again clearly distinct from either of them. Compared to the liquid, the bond orientational order is increased, but not quite crystalline yet, similar to the results for the pre-structured cloud observed during nucleation in $\mathrm{Ni}^{18}$ However, in contrast to the nucleation in an fcc material, the distributions of pre-structured atoms are shifted toward the bcc region. In particular, as the cluster size increases, the peak of the distribution (indicated by the red dot in Fig. 5) moves closer to bcc, that is, the orientational order in the pre-structured state continues to strengthen. While the density remains constant, the orientational order increases during nucleation and growth, thus promoting the formation of the bcc phase. The location of the distribution of the pre-ordered state on the $\bar{q}_{4}-\bar{q}_{6}$-map indicates that the selection of the final bulk polymorph is already triggered in the very early stages of nucleation by the structural features of the precursor.

\section{Voronoi polyhedra}

In addition to the bond orientational order, we analyze the local coordination around each atom by determining the corresponding Voronoi polyhedra (VP). The VP are characterized by a quartet of integers $\left\langle n_{3}, n_{4}, n_{5}, n_{6}\right\rangle$, where $n_{i}$ denotes the number of faces with $i$ vertices. ${ }^{52,53}$ In general, a single Voronoi polyhedron around an atom is not unique with respect to a specific crystal structure or phase, but each structure has a specific distribution of VP that acts 
(a)

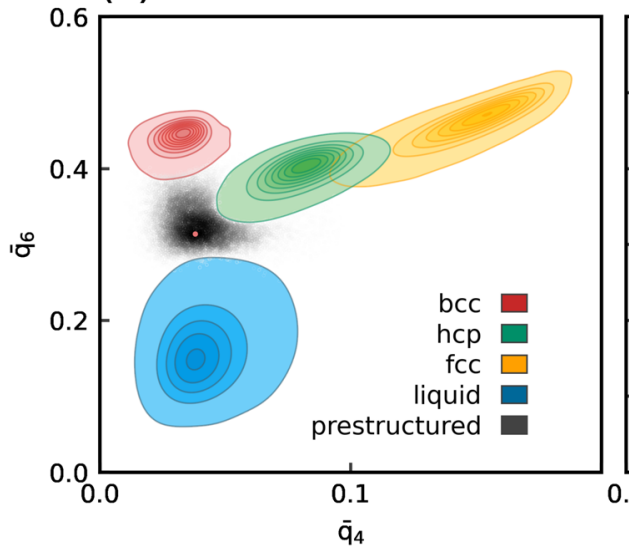

(b)

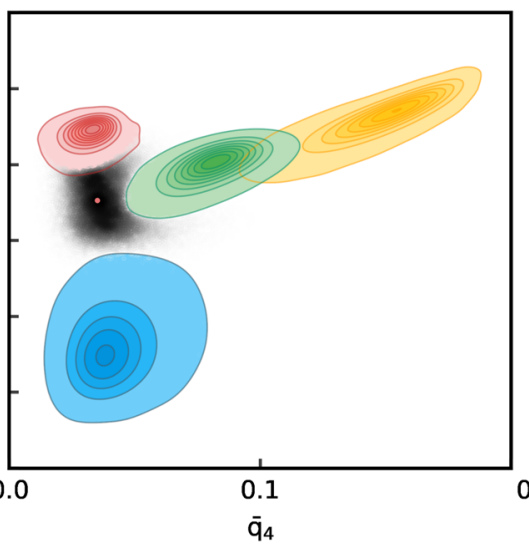

(c)

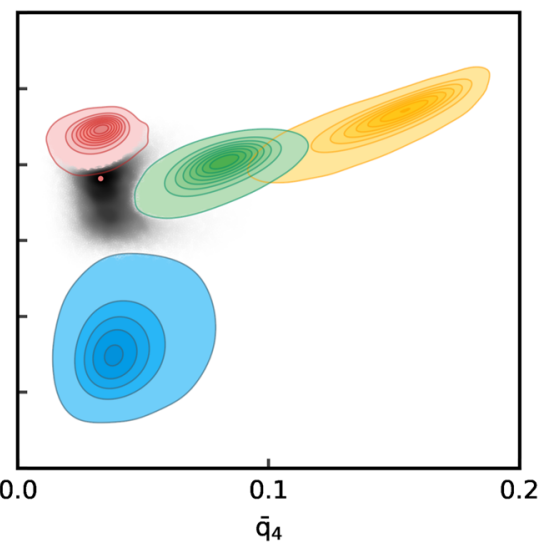

FIG. 5. Distributions of pre-structured particles (black) on the $\bar{q}_{4}-\bar{q}_{6}$-map for (a) pre-critical, $n_{s}=30$, (b) critical, $n_{s}^{*}=172$, and (c) post-critical, $n_{s}=370$ (right) clusters. Distributions of bulk bcc (red), hcp (green), fcc (yellow), and liquid (blue) are also shown. Regions of higher probability are indicated with a darker shade of the color. The peak of the distribution of pre-structured particle (red dot) shifts from $(0.038,0.314)$ in the pre-critical state to $(0.035,0.353)$ in the critical cluster and to $(0.033,0.382)$ for post-critical clusters.

as fingerprint. The most common VP that are found during nucleation and growth of bcc can be categorized broadly into four groups: $\langle 00120\rangle$ for a perfect icosahedron, $\langle 0110 x\rangle$ for distorted icosahedra, $\langle 0608\rangle$ for bcc, and a wide range of polyhedra of coordination numbers of 13 and 14 for distorted bcc.

In Fig. 6, we compare the distribution of VP at $20 \%$ undercooling extracted from 100 configurations in the liquid, the prestructured cloud at the critical nucleus size, and in bulk bcc. In the case of undercooled liquid, we focus on the inherent structure and relax the corresponding configurations until all forces are below
$10^{-4} \mathrm{eV} / \AA$ A before determining the VP. For the pre-structured region and the solid, we calculate the VP directly from the configurations. Additionally, we do not consider faces that contribute less than $1 \%$ to the total area in the classification of the VP. ${ }^{54}$

In the supercooled liquid, a very broad distribution of VP is found. The ten most common ones include the perfect icosahedron (dark blue in Fig. 6), a number of distorted icosahedra (light blue), and a few distorted bcc polyhedra (light red), similar to the findings in $\mathrm{Fe}^{34}$ and $\mathrm{Zr}^{36}$ In bulk bcc, mostly $\left\langle\begin{array}{llll}0 & 6 & 0 & 8\end{array}\right\rangle \mathrm{VP}$ (dark red) with minor amounts of distorted bcc polyhedra are observed. The (a)

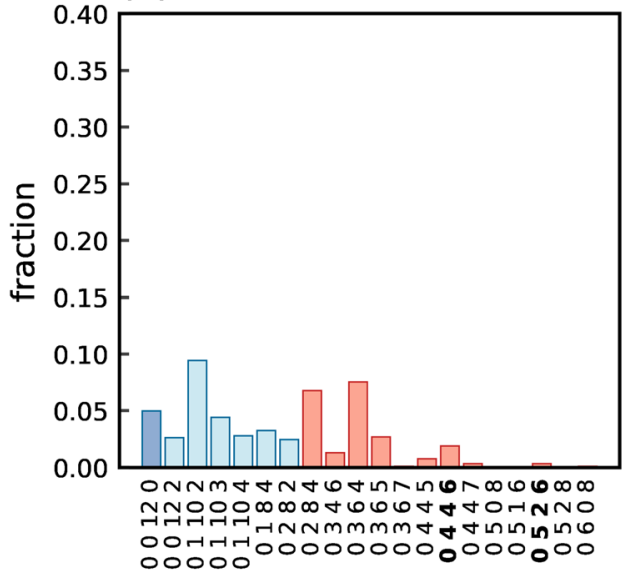

(b)

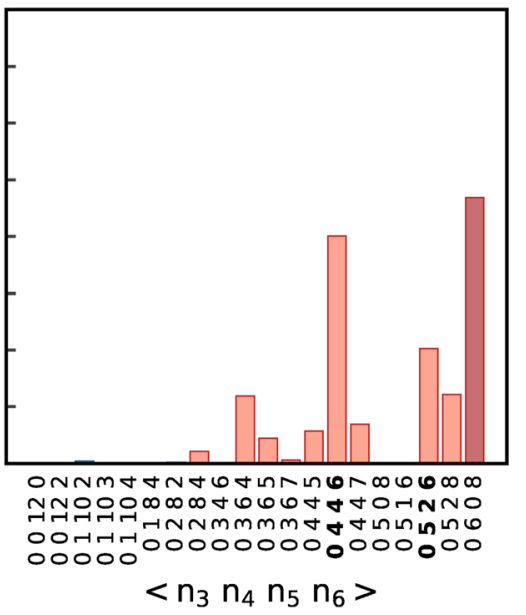

(c)

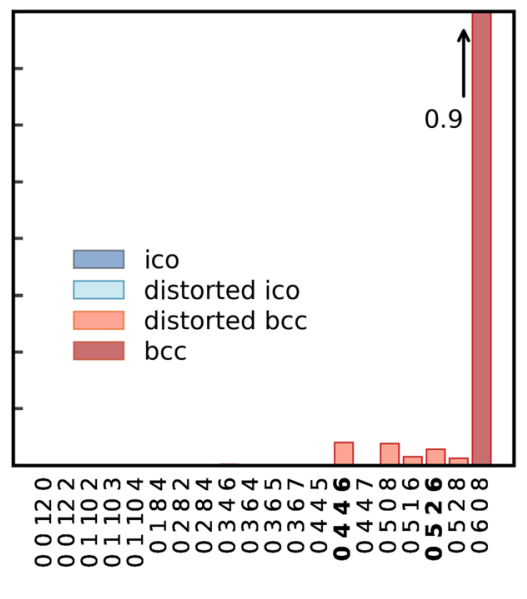

FIG. 6. Distribution of Voronoi polyhedra for (a) liquid, (b) pre-structured liquid, and (c) solid bcc at $20 \%$ undercooling. The distributions are shown for VP that include the top ten polyhedra most commonly found in the liquid, pre-ordered region, and solid. Icosahedral polyhedra are shown in dark blue, distorted icosahedral polyhedra are in light blue, distorted bcc and bcc are in light red and red, respectively. The most common distorted bcc polyhedra, $\langle 0446\rangle$ and $\langle 0526\rangle$ are highlighted in bold. $\langle 0608\rangle$ polyhedra in bcc peaks at a value of 0.9 . 
distribution of VP in the pre-structured liquid features mostly distorted bcc polyhedra, whereas icosahedra and distorted icosahedra disappear. Thus, coming from the liquid, a clear shift of the VP distribution takes place for the pre-ordered atoms. This corroborates the fact that the local structural environments in the pre-ordered cloud are very different from liquid. Furthermore, the VP in the pre-structured liquid show a clear tendency toward bcc ordering. Polyhedra of the type $\langle 0608\rangle$, although distorted as compared to the ones in perfect bulk bcc, appear frequently in the pre-structured liquid. The other two most common polyhedra of type $\langle 0446\rangle$ and $\left\langle\begin{array}{llll}0 & 5 & 2 & 6\end{array}\right\rangle$ (bold labels in Fig. 6) have previously been suggested as primary precursors for the formation of bcc Fe during quenching. ${ }^{33}$ Likewise, these VP are dominant in the distribution for the prestructured atoms, but do not play a major role in either the liquid or bulk bcc.

The analysis of the VP suggests that the pre-ordered liquid provides a precursor for the emergence of a specific bulk polymorph. Icosahedral motifs that generally hamper the formation of crystalline phases are reduced and local atomic environments with higher bond orientational order already reflect characteristic features of the final bulk phase. This indicates that in metallic systems, polymorph selection takes place in the very early stages of nucleation during the formation of the precursor zones.

In the range of undercoolings we have considered, from $20 \%$ to $25 \%$, the structural characteristics of the pre-ordered liquid are very similar. The distribution of $\mathrm{VP}$ for the pre-structured atoms shows a small decrease in the bcc polyhedron $\langle 0608\rangle$ from $34 \%$ to $27 \%$ and respective increase in the two most important, distorted bcc polyhedra $\langle 0446\rangle$ and $\left\langle\begin{array}{lll}0 & 5 & 2\end{array} 6\right\rangle$ with increasing undercooling. The slightly reduced structural ordering at larger undercoolings stems from the faster nucleation kinetics, as the atoms have less time to arrange into the thermodynamically preferred state.

\section{Influence of interatomic potentials on nucleation mechanism}

Due to the computational cost of TIS simulations, it remains still unfeasible to combine them with energies and forces based on electronic structure calculations. On the other hand, empirical interatomic potentials are computationally efficient, but are often developed to reproduce specific reference properties, with a particular focus on crystalline bulk phases, and, hence, might suffer from limited transferability. ${ }^{55}$ To evaluate the sensitivity of our results to the specific empirical potential used here, we have performed additional simulations with two further empirical potentials for Mo, a Finnis-Sinclair (FS), ${ }^{56,57}$ and a modified EAM (MEAM) ${ }^{58}$ potential.

The simulation results obtained with the FS potential are very similar to the EAM ones. The melting temperature is somewhat lower, $T_{m}=2850 \mathrm{~K}$, and consequently the absolute values of the nucleation barriers and rates differ, but show the same trends and are in very good qualitative agreement (see the supplementary material for details).

The nucleation mechanism is the same in the two potentials, including the formation of a pre-ordered region in the liquid with the subsequent emergence of a bcc crystalline core. Furthermore, the structural characteristics of the precursor are in excellent agreement, the distribution of VP obtained with the FS potential (shown in Fig. S1 in the supplementary material) is nearly identical to the one shown in Fig. 6. Clearly, the results obtained with the FS potential are very much comparable and lead to the same assessment of the nucleation mechanism in Mo.

With the MEAM potential for Mo used in this work, nucleation and growth of the bcc bulk from the undercooled liquid could not be observed. Instead, an amorphous phase forms akin to a glassy state. The failure of the employed MEAM potential to solidify into the crystalline bcc phase can be understood from the analysis of the undercooled liquid. In Fig. 7, the distribution of VP is shown for the liquid at $20 \%$ undercooling using the FS and MEAM potential. While the distribution obtained with the FS potential is essentially the same as for the EAM potential in Fig. 6, the distribution using the MEAM potential is clearly different. The amount of icosahedra (dark blue) and distorted icosahedra (light blue) increases and the number of distorted bcc polyhedra (light red) decreases. Specifically, the absence of the distorted bcc polyhedra impedes the formation of pre-structured liquid and pushes the system toward amorphization. The MEAM potential does not accurately represent the heterogeneous nature of the undercooled liquid, which is decisive in describing the nucleation process. ${ }^{59}$ A reason for this (a)

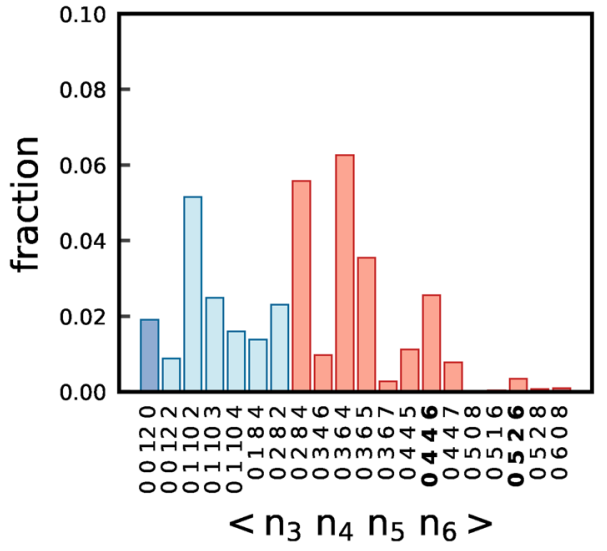

(b)

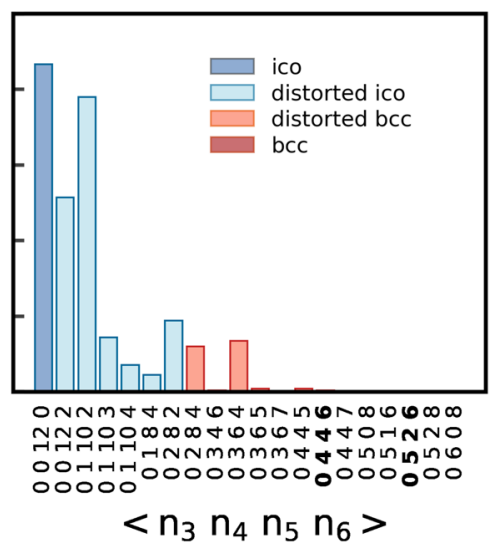

FIG. 7. Distributions of Voronoi polyhedra for liquid at $20 \%$ undercooling using a (a) FS and (b) MEAM potentials. Icosahedral polyhedra are shown in dark blue, distorted icosahedral polyhedra are in light blue, and distorted bcc are in light red. 
could be a too strong contribution of the angular term in this MEAM potential.

Comparing the three potentials, EAM, FS, and MEAM, it becomes apparent that a suitable empirical potential should be able to correctly capture the structural features of both, the liquid and solid, phases of a material. If this is fulfilled, the overall nucleation mechanism appears to be less sensitive to a particular empirical potential.

\section{CONCLUSIONS}

We analyze the nucleation mechanism during solidification in Mo, which proceeds via two steps: the initial formation of preordered regions in the supercooled liquid and a subsequent formation of the crystalline bcc bulk phase within these regions. The pre-ordered liquid region has a long lifetime and surrounds the bcc phase in the growing cluster even beyond the critical nucleus size. The increase in the bond-orientational order within this region creates a precursor that reduces the interfacial free energy by providing a diffuse interface between the liquid and crystalline core of the growing nucleus, thus facilitating the formation of the bulk phase.

While the density within the pre-ordered liquid remains constant for the growing clusters, the bond-orientational order increases, showing that this is the decisive factor that triggers the nucleation process. Furthermore, the analysis of the structural composition of the pre-ordered region reveals clear differences from the liquid and solid phases, showing no icosahedral structures and a strong tendency toward (distorted) bcc like environments. The structure of the pre-ordered region, thus, promotes the formation of a specific bulk structure and is key in the selection of the final polymorph.

The structural heterogeneities in the undercooled liquid are very important in the crystallization process. Indeed, we find that the nucleation mechanism strongly depends on the ability of the interatomic potential to correctly capture the structural hallmarks of the liquid. The complete absence of distorted bcc like environments and an abundance of icosahedral structures (as predicted by the employed MEAM potential) inhibit the formation of the precursor and, consequently, promote the formation of amorphous structures instead of crystalline bulk phases. This finding agrees well with the recent work showing that an increase in the structural difference between liquid and crystal enhances the glass-forming ability and is linked with the suppression of crystal precursors.

Compared to other metallic systems, such as fcc $\mathrm{Ni}^{18}$ and $\mathrm{Al},{ }^{17}$ the overall nucleation mechanism is very similar and appears to be generally valid, in particular the formation of a precursor of high orientational order followed by the emergence of the crystalline phase within the cluster core. The structure of the pre-ordered liquid is, however, inherently different for these systems, strongly indicating the key role of precursors in the selection of the final polymorph at the very early stages of nucleation. This also implies that controlling the formation of specific bulk polymorph through seeding or environmental conditions is closely associated with controlling the structure and formation of the precursor zones in the supercooled liquid.

\section{SUPPLEMENTARY MATERIAL}

See the supplementary material for details on the TIS setup, on nucleation barriers and rates, differences between pre-structuring and solid-liquid interface, and on the comparison with the FS potential, which includes Refs. 60 and 61.

\section{ACKNOWLEDGMENTS}

S.M. acknowledges a scholarship from the International Max Planck Research School for Interface Controlled Materials for Energy Conversion. We acknowledge the financial support from the Deutsche Forschungsgemeinschaft (DFG) through Project Nos. 262052203 and 211503459 (C2 of the collaborative research center SFB/TR 103). The authors acknowledge computing time by the Center for Interface-Dominated High Performance Materials (ZGH, Ruhr-Universität Bochum).

\section{DATA AVAILABILITY}

The data that support the findings of this study are available from the corresponding author upon reasonable request.

\section{REFERENCES}

${ }^{1}$ G. C. Sosso, J. Chen, S. J. Cox, M. Fitzner, P. Pedevilla, A. Zen, and A. Michaelides, Chem. Rev. 116, 7078 (2016).

${ }^{2}$ R. Becker and W. Döring, Ann. Phys. 416, 719 (1935).

${ }^{3}$ K. Binder, Rep. Prog. Phys. 50, 783 (1987).

${ }^{4}$ M. Horsch, J. Vrabec, and H. Hasse, Phys. Rev. E 78, 011603 (2008).

${ }^{5}$ S. Prestipino, A. Laio, and E. Tosatti, J. Chem. Phys. 140, 094501 (2014).

${ }^{6}$ L. Gránásy, Mater. Sci. Forum 215-216, 451 (1996).

${ }^{7}$ S. Prestipino, A. Laio, and E. Tosatti, Phys. Rev. Lett. 108, 225701 (2012).

${ }^{8}$ W. C. Swope and H. C. Andersen, Phys. Rev. B 41, 7042 (1990).

${ }^{9}$ P. R. ten Wolde, M. J. Ruiz-Montero, and D. Frenkel, Phys. Rev. Lett. 75, 2714 (1995).

${ }^{10}$ P. R. ten Wolde, M. J. Ruiz-Montero, and D. Frenkel, J. Chem. Phys. 104, 9932 (1996).

${ }^{11}$ S. Auer and D. Frenkel, Nature 409, 1020 (2001).

${ }^{12}$ T. Schilling, H. J. Schöpe, M. Oettel, G. Opletal, and I. Snook, Phys. Rev. Lett. 105, 025701 (2010).

${ }^{13}$ T. Kawasaki and H. Tanaka, Proc. Natl. Acad. Sci. U. S. A. 108, 6335 (2011).

${ }^{14}$ W. Lechner, C. Dellago, and P. G. Bolhuis, J. Chem. Phys. 135, 154110 (2011).

${ }^{15}$ J. Russo and H. Tanaka, Sci. Rep. 2, 505 (2012).

${ }^{16}$ A. Lederer, M. Franke, and H. J. Schöpe, Eur. Phys. J.: Spec. Top. 223, 389 (2014).

${ }^{17}$ C. Desgranges and J. Delhommelle, J. Chem. Phys. 127, 144509 (2007).

${ }^{18}$ G. Díaz Leines, R. Drautz, and J. Rogal, J. Chem. Phys. 146, 154702 (2017).

${ }^{19}$ G. Díaz Leines and J. Rogal, J. Phys. Chem. B 122, 10934 (2018).

${ }^{20}$ J. Russo and H. Tanaka, Soft Matter 8, 4206 (2012).

${ }^{21}$ J. F. Lutsko and G. Nicolis, Phys. Rev. Lett. 96, 046102 (2006).

${ }^{22}$ P. G. Vekilov, Cryst. Growth Des. 4, 671 (2004).

${ }^{23}$ P. R. t. Wolde and D. Frenkel, Science 277, 1975 (1997).

${ }^{24}$ P. R. ten Wolde and D. Frenkel, Phys. Chem. Chem. Phys. 1, 2191 (1999).

${ }^{25}$ J. Russo, F. Romano, and H. Tanaka, Phys. Rev. X 8, 021040 (2018).

${ }^{26}$ U. Gasser, A. Schofield, and D. A. Weitz, J. Phys.: Condens. Matter 15, S375 (2003).

${ }^{27}$ N. Jakse and A. Pasturel, Phys. Rev. Lett. 91, 195501 (2003).

${ }^{28}$ N. Jakse and A. Pasturel, J. Chem. Phys. 120, 6124 (2004).

${ }^{29}$ F. Puosi and A. Pasturel, Phys. Rev. Mater. 3, 023402 (2019).

${ }^{30}$ C. Dellago, P. Bolhuis, and P. L. Geissler, Adv. Chem. Phys. 123, 1 (2002). 
${ }^{31}$ D. Moroni, T. S. Van Erp, and P. G. Bolhuis, Phys. Rev. E 71, 056709 (2005).

${ }^{32}$ A. Garner, Can. Metall. Q. 16, 48 (1977).

${ }^{33}$ S. P. Pan, S. D. Feng, J. W. Qiao, W. M. Wang, and J. Y. Qin, Sci. Rep. 5, 16956 (2015).

${ }^{34}$ J. Wang, Q. Zhang, S. Tang, W. Zhou, Z. Wang, Y. Wang, and J. Li, Phys. Chem. Chem. Phys. 21, 4122 (2018)

${ }^{35}$ R. Li, Y. Wu, and J. Xiao, J. Chem. Phys. 140, 034503 (2014).

${ }^{36} \mathrm{~S}$. Wu, X. W. Fang, S. Y. Wang, C. Z. Wang, Y. X. Yao, K. M. Ho, Z. J. Ding, and L. Y. Chen, J. Appl, Phys. 110, 103518 (2011).

${ }^{37}$ T. S. Van Erp, D. Moroni, and P. G. Bolhuis, J. Chem. Phys. 118, 7762 (2003).

${ }^{38}$ J. Rogal, W. Lechner, J. Juraszek, B. Ensing, and P. G. Bolhuis, J. Chem. Phys. 133, 174109 (2010).

${ }^{39}$ P. G. Bolhuis and W. Lechner, J. Stat. Phys. 145, 841 (2011).

${ }^{40}$ S. Plimpton, J. Comput. Phys. 117, 1 (1995).

${ }^{41}$ X. W. Zhou, R. A. Johnson, and H. N. G. Wadley, Phys. Rev. B 69, 144113 (2004).

${ }^{42}$ A. B. Belonoshko, N. V. Skorodumova, A. Rosengren, and B. Johansson, Phys. Rev. B 73, 012201 (2006).

${ }^{43}$ The TPS-wrapper, a PYTHON-based wrapper to perform transition path sampling coupled with several molecular dynamics drivers, is developed at ICAMS, Ruhr-Universität Bochum. The package can be requested from the corresponding authors via email.
${ }^{44}$ T. S. Van Erp, Phys. Rev. Lett. 98, 268301 (2007).

${ }^{45}$ P. J. Steinhardt, D. R. Nelson, and M. Ronchetti, Phys. Rev. B 28, 784 (1983).

${ }^{46}$ S. Auer and D. Frenkel, Adv. Polym. Sci. 173, 149 (2005).

${ }^{47}$ J. Bokeloh, R. E. Rozas, J. Horbach, and G. Wilde, Phys. Rev. Lett. 107, 145701 (2011).

${ }^{48}$ W. Lechner and C. Dellago, J. Chem. Phys. 129, 114707 (2008).

${ }^{49}$ S. Menon, G. Díaz Leines, and J. Rogal, J. Open Source Software 4, 1824 (2019).

${ }^{50}$ H. Tanaka, H. Tong, R. Shi, and J. Russo, Nat. Rev. Phys. 1, 333 (2019).

${ }^{51}$ C. H. Rycroft, Chaos 19, 041111 (2009).

${ }^{52}$ J. L. Finney, Proc. R. Soc. London, Ser. A 319, 479 (1970).

${ }^{53}$ M. Tanemura, Y. Hiwatari, H. Matsuda, T. Ogawa, N. Ogita, and A. Ueda, Prog. Theor. Phys. 58, 1079 (1977).

${ }^{54}$ A. Stukowski, Modell. Simul. Mater. Sci. Eng. 20, 045021 (2012).

${ }^{55}$ Y. Lysogorskiy, T. Hammerschmidt, J. Janssen, J. Neugebauer, and R. Drautz, Modell. Simul. Mater. Sci. Eng. 27, 025007 (2019).

${ }^{56}$ M. W. Finnis and J. E. Sinclair, Philos. Mag. A 50, 45 (1984).

${ }^{57}$ G. J. Ackland and R. Thetford, Philos. Mag. A 56, 15 (1987).

${ }^{58}$ B.-J. Lee, M. I. Baskes, H. Kim, and Y. Koo Cho, Phys. Rev. B 64, 184102 (2001).

${ }^{59}$ J. Russo and H. Tanaka, J. Chem. Phys. 145, 211801 (2016).

${ }^{60}$ D. Turnbull, J. Appl. Phys. 21, 1022 (1950).

${ }^{61}$ F. Spaepen, Solid State Phys. 47, 1 (1994). 